integrationspolitische Fragen wie die Revision der EU-Verträge umstritten. Der innenpolitisch sonst nicht etablierte Konflikt zwischen supranationaler Integration und nationalstaatlicher Autonomie wird also ebenfalls in den Debatten aufgenommen. Europapolitische Kontroversen sind also nicht ausschließlich als Reproduktion der innenpolitischen Auseinandersetzung anzusehen, sondern bringen durchaus neuartige Fragen und Kontroversen ins Parlament ein. Interessant ist dabei die unterschiedliche Logik der supranationalen und innenpolitischen Konfliktebene: Während die nationale Europapolitik wie erwartet innerhalb eines Antagonismus zwischen Regierung und Opposition debattiert wird, zeigen sich gegenüber supranationalen Themen andere Positionsmuster, die diesen Gegensatz überbrücken. Eine zunehmende Politisierung supranationaler Themen könnte etablierte innenpolitische Konfliktmuster also zunehmend in Frage stellen.

Abschließend erscheint noch eine Bemerkung zum hier gewählten quantitativen Verfahren angebracht. Als Vorzug ist festzuhalten, dass es die effiziente Auswertung einer großen Textmenge und die vergleichende Bewertung eines großen Themenspektrums erlaubt. Eine vertiefende Betrachtung der inhaltlichen Struktur der Parteipositionen und der Interaktion der Sprecher in parlamentarischen Debatten kann aber nur im Rahmen einer qualitativen Inhaltsanalyse geleistet werden. Diese erscheint damit als sinnvoller und wünschenswerter nächster Schritt.

\title{
Die Initiativtätigkeit des Bundesrates im Lichte der Parteipolitisierungsthese
}

\author{
Isabella Harle und Christian Stecker*
}

Seit Bestehen des Bundesrates beschäftigt seine Stellung zwischen Parteipolitik und Vertretung der Länderinteressen Politikwissenschaft und Öffentlichkeit. ${ }^{1}$ Fundierte Antworten sind dabei insbesondere aus normativer Perspektive relevant. Der Bundesrat soll nach herrschender Auffassung die Vertretung der Länder in der Gesetzgebung ermöglichen ${ }^{2}$ - eine Parteipolitisierung der Länderkammer widerspräche dieser Aufgabe. ${ }^{3}$ Zudem wird das

* Wir danken Johannes Kuhn für hilfreiche Kommentare und seine Unterstützung bei der Fertigstellung des Manuskriptes.

1 Vgl. Gerhard Lehmbruch, Parteienwettbewerb im Bundesstaat, Wiesbaden 2000.

2 Vgl. Hans Herbert von Arnim, Strukturprobleme des Parteienstaates, in: APuZ, B 16 (2000), S. $30-38$.

3 Dagegen Roland Johne, Bundesrat und parlamentarische Demokratie. Die Länderkammer zwischen Entscheidungshemmnis und notwendigem Korrektiv in der Gesetzgebung, in: APuZ, B 50/51 (2004), S. 10 - 17. Allerdings zeigen Analysen zum Entstehungsprozess des Bundesrates, dass ihm von einigen Akteuren auch eine Rolle als „Widerlager“ in der Parteipolitik, im Sinne einer „Hemmung“" des Bundestages, zugedacht wurde. Vgl. Karlheinz Niclauß, Parlament und Zweite Kammer in der westdeutschen Verfassungsdiskussion von 1946 bis zum Parlamentarischen Rat, in: ZParl, 39. Jg. (2008), H. 3, S. 595 - 611, S. 610. 
kompetitive Verhalten von Parteien im Bundesrat ${ }^{4}$ mit negativen Folgen für das gesamte politische System assoziiert. Es führe ,immer wieder zu Verzögerungen oder sogar Verhinderung wichtiger Gesetzesvorhaben" schwere insgesamt das Regieren auf Bundesebene. ${ }^{6}$

Zahlreiche politikwissenschaftliche Studien haben bereits versucht, Vorhandensein, Umfang und Konsequenz einer Parteipolitisierung des Bundesrates einzuschätzen. Erhellende Einblicke aus qualitativen Fallstudien ${ }^{7}$ konnten in jüngster Zeit durch quantitative Analysen auf Basis der Gesetzgebungsstatistik ${ }^{8}$ ergänzt werden. Ihre gemeinsame Grundidee ist, dass man einer Parteipolitisierung auf die Spur kommen kann, indem der Gesetzgebungsprozess in Abhängigkeit unterschiedlicher Mehrheitsverhältnisse im Bundesrat untersucht wird. Stimmte ein oppositionskontrollierter Bundesrat beispielsweise häufiger gegen Gesetzentwürfe der Bundestagsmehrheit, spräche dies für einen „Einbruch“ (bundes-)parteipolitischer Gegensätze in die Länderkammer. ${ }^{9}$ Dominieren hingegen Länderinteressen, müsste der Gang der Gesetzgebung von der parteipolitischen Zusammensetzung im Bundesrat weitgehend unberührt bleiben. Sein Entscheidungsverhalten sollte dann vornehmlich durch horizontale Konflikte zwischen den Ländern (zum Beispiel Länderfinanzausgleich) und vertikale Bund-Länder-Konflikte strukturiert werden. ${ }^{10}$

Resümiert man die Untersuchungen auf Basis der Gesetzgebungsstatistik, zeigt sich, dass sie vorrangig auf das Ende des Gesetzgebungsprozesses gerichtet sind und somit die reaktive

4 Siehe für eine klare begriffliche Abgrenzung Thomas Bräuninger / Steffen Ganghof, Parteienwettbewerb im Zweikammersystem, in: Steffen Ganghof I Philip Manow (Hrsg.), Mechanismen der Politik. Strategische Interaktion im deutschen Regierungssystem, Frankfurt am Main 2005, S. 149 -181 .

5 BT-Drs. 16/2010, S. 3.

6 Vgl. Gerd Langguth, Machtverteilung und Machtverschränkung in Deutschland, in: APuZ, B 6 (2000), S. 3 - 11, S. 4 f.

7 Vgl. Sven Leunig, „Öl“ oder "Sand“ im Getriebe? Der Einfluss der Parteipolitik auf den Bundesrat als Veto-Spieler im Gesetzgebungsprozess, in: ZParl, 34. Jg. (2003), H. 4, S. 778 - 791; Thomas Saalfeld, Conflict and Consensus in Germany's Bi-cameral System: A Case Study of the Passage of the Agenda 2010, in: Debatte, 14. Jg. (2006), H. 3, S. 247 - 269; Reimut Zohlnhöfer, Die große Steuerreform 1998/99: Ein Lehrstück für Politikentwicklung bei Parteienwettbewerb im Bundesstaat, in: ZParl, 30. Jg. (1999), H. 2, S. 326 - 345; Wolfgang Renzsch, Bundesstaat oder Parteienstaat: Überlegungen zu Entscheidungsprozessen im Spannungsfeld von föderaler Konsensbildung und parlamentarischem Wettbewerb in Deutschland, in: Everhard Holtmann / Helmut Voelzkow (Hrsg.), Zwischen Wettbewerbsdemokratie und Verhandlungsdemokratie. Analysen zum Regierungssystem der Bundesrepublik Deutschland, Opladen 2000, S. 53 - 78.

8 Vgl. Thomas König / Thomas Bräuninger, Gesetzgebung im Föderalismus, Speyer 2005; Thomas Bräuninger / Thomas Gschwend / Susumu Shikano, Sachpolitik oder Parteipolitik? Eine Bestimmung des Parteidrucks im Bundesrat mittels bayesianischer Methoden, in: PVS, 51. Jg. (2010), H. 2, S. 223 - 249; Philip Manow / Simone Burkhart, Legislative Self-Restraint under Divided Government in Germany, 1976-2002, in: Legislative Studies Quarterly, 32. Jg. (2007), H. 2, S. 167 191; Bernhard Miller / Christian Stecker, Consensus by Default? Interaction of Government and Opposition Parties in the Committees of the German Bundestag, in: German Politics, 17. Jg. (2008), H. 3, S. 307 - 324; Gerd Strohmeier, Der Bundesrat: Vertretung der Länder oder Instrument der Parteien?, in: ZParl, 35. Jg. (2004), H. 4, S. 717 - 731; Werner Reutter, Struktur und Dauer der Gesetzgebungsverfahren des Bundes, in: ZParl, 38. Jg. (2007), H. 2, S. 299 - 315.

9 Differenzierter Simone Burkhart / Philip Manow, Kompromiss und Konflikt im parteipolitisierten Föderalismus der Bundesrepublik Deutschland, in: ZfP, 16. Jg. (2006), H. 3, S. 807 - 824.

10 Vgl. Sven Leunig, a.a.O. (Fn. 7), S. 782. 
Rolle des Bundesrates beleuchten. ${ }^{11}$ Vernachlässigt wird dabei seine Initiativtätigkeit. Die vorliegende Analyse schließt diese Lücke. Sie betrachtet die Gesetzesinitiativen aus der Mitte des Bundesrates und nimmt so seine proaktive Rolle in den Blick. Damit folgt sie dem Desiderat, die Beteiligung der Länderkammer im Gesetzgebungsprozess möglichst vollständig abzubilden. ${ }^{12}$

\section{Theoretische Überlegungen: Der Bundesrat als oppositionelles Instrument der Gesetzesinitiative?}

Gemäß Art. 50 GG wirken die Länder durch den Bundesrat an der Gesetzgebung und Verwaltung des Bundes mit. Dazu gehört neben Einspruchs- und Zustimmungsrechten auch das Initiativrecht des Bundesrates (Art. 76 Abs. 1 GG). Jedes Land oder eine Gruppe von Ländern kann im Rahmen des allgemeinen Antragsrechts Gesetzesinitiativen einbringen. Der Bundesrat entscheidet mit absoluter Mehrheit seiner Stimmen über die Zuleitung dieser Vorlage an die Bundesregierung. ${ }^{13}$ Diese hat den Entwurf innerhalb von sechs Wochen dem Bundestag weiterzuleiten (Art. 76 Abs. 3 GG) und soll dabei ihre Auffassung darlegen. ${ }^{14}$ Allgemein gilt, dass "die Initiativfunktion als eine der herausragenden Steuerungsmöglichkeiten von Politik“ angesehen werden kann, da durch „die Gesetzesinitiative [...] die politische Agenda besetzt und das Programm umgesetzt werden [kann] "15.

Wäre der Bundesrat eine reine Vertretung von Länderinteressen sollten sich seine Gesetzesvorlagen vorrangig auf die Bund-Länder-Dimension beziehen. In diesem Bereich dürften die Landesvertreter besonders häufig ein gemeinsames Interesse formulieren können. Ebenso sollten Initiativen zu finden sein, in denen die Länder ihre Erfahrungen aus dem Vollzug von Gesetzen in den Gesetzgebungsprozess einbringen. Etwas seltener ist mit Vorlagen zu rechnen, die horizontale Land-Land-Konflikte thematisieren. Nur wenn eine Seite des Konfliktes über eine (absolute) Mehrheit in der Kammer verfügt, können diese Vorlagen der Bundesregierung zugeleitet werden.

Ist dagegen die parteipolitische Logik bestimmend, sollte die Initiativtätigkeit des Bundesrates auch durch (bundes-)parteipolitische Konflikte strukturiert werden beziehungsweise sich auf einer parteipolitischen Rechts-links-Achse abbilden lassen. ${ }^{16}$ Konkret müsste sich eine Parteipolitisierung offenbaren, wenn die oppositionelle Minderheit des Bundes-

11 Lediglich Gerd Strohmeier, a.a.O. (Fn. 8), betrachtet mit der Ablehnung von Bundesratsvorlagen im Bundestag ein früheres Stadium des Gesetzgebungsprozesses. Siehe auch Martin Brunner / Marc Debus, Between Programmatic Interests and Party Politics: The German Bundesrat in the Legislative Process, in: German Politics, 17. Jg. (2008), H. 3, S. $232-251$.

12 Vgl. Gerd Strohmeier, a.a.O. (Fn. 8), S. 718.

13 Vgl. Wolfgang Ismayr, Gesetzgebung im politischen System Deutschlands, in: ders. (Hrsg.), Gesetzgebung in Westeuropa. EU-Staaten und Europäische Union, Wiesbaden 2008, S. 383 - 430, S. 393.

14 Zum weiteren Verlauf siehe Manfred G. Schmidt, Das politische System Deutschlands, München 2007; Wolfgang Rudzio, Das politische System der Bundesrepublik Deutschland, Opladen 2006.

15 Thomas König / Thomas Bräuninger, a.a.O. (Fn. 8), S. 6.

16 Vgl. Thomas König / Thomas Bräuninger, Wie wichtig sind die Länder für die Politik der Bundesregierung bei Einspruchs- und Zustimmungsgesetzen?, in: ZParl, 28. Jg. (1997), H. 4, S. 605 628; Thomas Bräuninger / Thomas Gschwend / Susumu Shikano, a.a.O. (Fn. 8). 
tages über eine Mehrheit im Bundesrat verfügt. Diese könnte, unter der Voraussetzung parteipolitischer Geschlossenheit, das Initiativverhalten des Bundesrates diktieren. Zugespitzt formuliert, diente der Bundesrat der Opposition dann nicht nur als potentielles Blockadeinstrument ${ }^{17}$, sondern auch als Vehikel für Gesetzesinitiativen. Auf den ersten Blick scheint eine solche Nutzung des Bundesrates unnötig zu sein, denn im Bundestag kann jede Fraktion (beziehungsweise Abgeordnete in Fraktionsstärke), also auch die Opposition Gesetzesvorlagen initiieren. Formuliert als Initiative „ihrer“ Länder kann die Bundestagsopposition allerdings „mit dem politischen Gewicht der Ländervertretung oppositionelle Alternativ-entwürfe in die politische Willensbildung im Parlament" einbringen. ${ }^{18}$ Diese Vorlagen würden im Bundestag dann unter dem Etikett parteipolitisch neutraler, territorialer Inte-ressen beraten werden, was eine Ablehnung durch die Parlamentsmehrheit potentiell schwieriger macht. Denkbar ist auch, dass Bundesratsinitiativen der Opposition als „Verhandlungsmasse“ in bundespolitischen Konflikten genutzt werden. Für die Koordination derartig parteipolitisch motivierter Initiativen ${ }^{19}$ stehen den Landes- und Bundesparteiorganisationen umfangreiche Koordinationsmechanismen zur Verfügung. ${ }^{20}$

\section{Empirische Überprüfung einer Parteipolitisierung der Initiativtätigkeit}

\section{1. Überblick über Länderinitiativen im Bundesrat zwischen 1972 und 2005}

Grundlage dieser Untersuchung ist ein Datensatz, der aus den Informationen zum Stand der Gesetzgebung (GESTA) zwischen der 7. und 15. Wahlperiode (1972 bis 2005) ${ }^{21}$ gewonnen wurde. ${ }^{22}$ Er beinhaltet unter anderem alle 1.332 in diesem Zeitraum in den Bundesrat eingebrachten Gesetzesanträge der Länder.

Für knapp drei Viertel dieser Anträge zeichnete jeweils nur ein einzelnes Bundesland verantwortlich. Die übrigen Vorlagen wurden von Ländergruppen eingebracht. Abbildung 1 schlüsselt diese Initiativen nach antragstellenden Bundesländern auf. Dabei wird differen-

17 Vgl. Klaus Stüwe, Konflikt und Konsens im Bundesrat. Eine Bilanz (1949-2004), in: APuZ, B 50-51 (2004), S. 25 - 32.

18 Vgl. Jürgen Jekewitz, Art. 76 GG, in: Erhard Denninger / Wolfgang Hoffmann-Riem / Hans-Peter Schneider / Ekkehart Stein (Hrsg.), Kommentar zum Grundgesetz für die Bundesrepublik Deutschland (Alternativkommentar), Neuwied 2001, S. 22.

19 Vgl. Wolfgang Ismayr, a.a.O. (Fn. 13), S. 393 f.

20 Vgl. Uwe Leonardy, Parteien im Föderalismus der Bundesrepublik Deutschland. Scharniere zwischen Staat und Politik, in: ZParl, 33. Jg. (2002), H. 1, S. 181 - 195. Wie Wolfgang Renzsch, a.a.O. (Fn. 7), zeigt, können durch diese Koordinationsmechanismen föderale Konfliktlagen häufig einer innerparteilichen Lösung zugeführt werden.

21 Es wäre wünschenswert, die empirische Überprüfung bis mindestens 1969 auszudehnen, als mit der Regierungsübernahme der sozial-liberalen Koalition die Polarisierung des Parteienwettbewerbs zunahm und auch deutlich auf den Bundesrat einwirkte, siehe Gerhard Lehmbruch, a.a.O. (Fn. 1), S. 141 - 148. Leider wird der Stand der Gesetzgebung erst seit 1972 systematisch aufbereitet. Weiter zurückliegende Vorgänge müssten über die Analyse gedruckter Sachregister rekonstruiert werden. Der enorme Erhebungsaufwand wäre zweifelsfrei in vielerlei Hinsicht lohnenswert - er muss aber einem umfangreicheren Projekt vorbehalten bleiben.

22 Vgl. Bernhard Miller / Christian Stecker, a.a.O. (Fn. 8); Simone Burkhart / Philip Manow, Kompromiss und Konflikt im parteipolitisierten Föderalismus der Bundesrepublik Deutschland, in: ZfP, 16. Jg. (2006), H. 3, S. 807 - 824. 


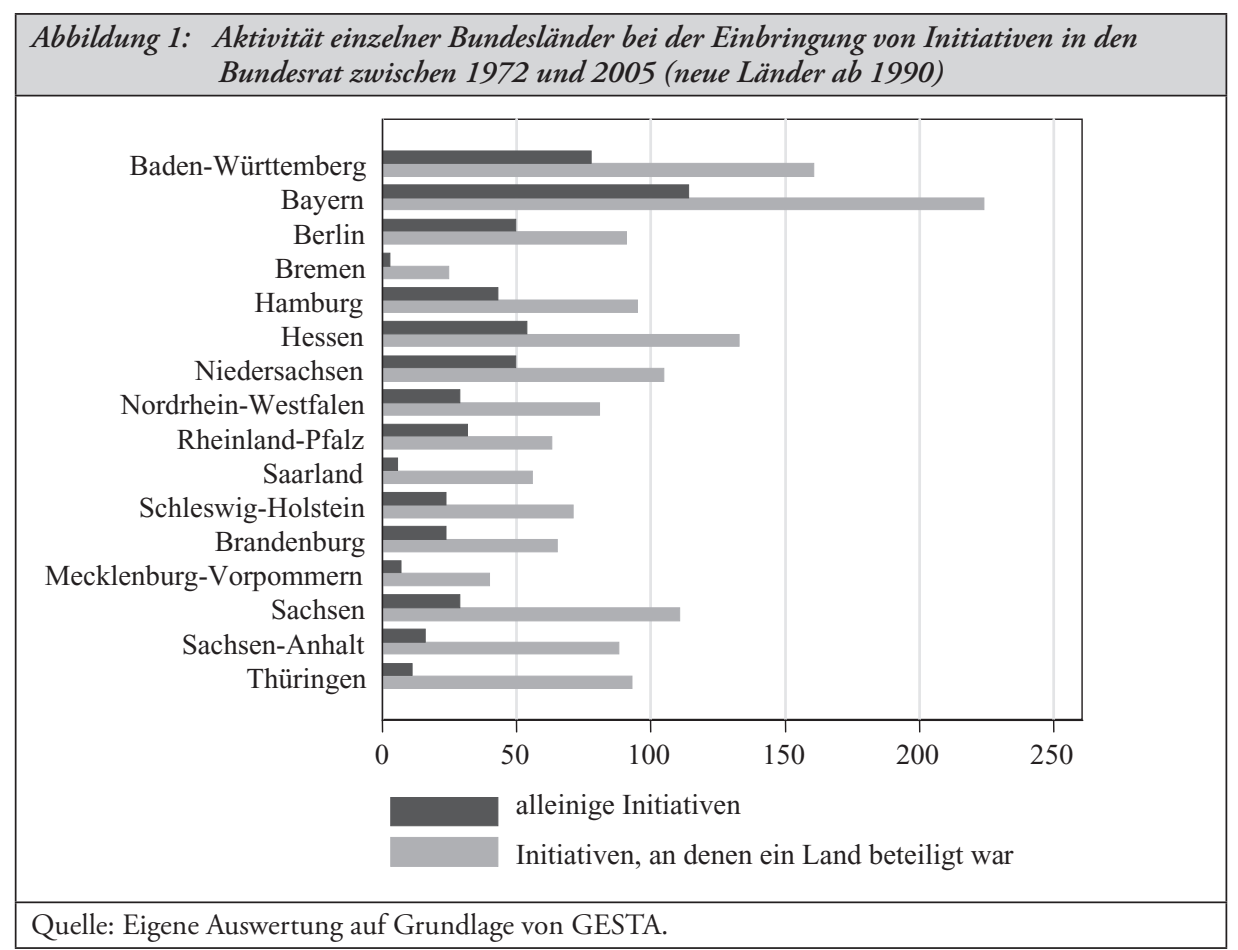

ziert nach alleinigen Initiativen eines Landes und der Gesamtzahl der Initiativen, an denen ein Bundesland beteiligt war. Es zeigt sich, dass Bayern, Baden-Württemberg und Hessen besonders rege von ihrem Antragsrecht Gebrauch machten. Bayern startete 114 alleinige Initiativen im Bundesrat und war mit weiteren 110 Gruppenanträgen an insgesamt 224 Vorlagen beteiligt. ${ }^{23}$ Berücksichtigt man nur die Zeit nach der Wiedervereinigung liegen auch die ostdeutschen Bundesländer Brandenburg, Sachsen, Sachsen-Anhalt und Thüringen bei der Antragstätigkeit im oberen Mittelfeld.

Insgesamt wurde nur bei 13 Prozent (172) der 1.332 Vorlagen eine Einbringung in den Bundestag abgelehnt. 36 Prozent wurden anderweitig erledigt, das heißt entweder zurückgezogen, mit anderen Vorlagen vereint oder nicht weiter im Bundesrat beraten (siehe Abbildung 2). Die drei abgesetzten Teile der Grafik auf der linken Seite zeigen den Verlauf der insgesamt 685 (gut 51 Prozent aller Länderinitiativen) Vorlagen, die an den Bundestag weitergeleitet wurden. Zehn Prozent (bezogen auf alle Länderinitiativen) wurden vom Bundestag abgelehnt und 28 Prozent anderweitig erledigt. Lediglich 14 Prozent aller Länderinitiativen gelang der Weg ins Bundesgesetzblatt. Höchstwahrscheinlich fanden wesentlich mehr Länderinitiativen implizit in der Gesetzgebung ihren Niederschlag. Dies kann mit den vorliegenden Daten jedoch nicht nachvollzogen werden.

23 Es wäre interessant zu prüfen, inwiefern diese Vorlagen dazu dienten, den bundespolitischen Anspruch der CSU zu untermauern. Denkbar ist ebenso, dass die Häufigkeit von Einparteimehrheitsregierungen in Bayern und Baden-Württemberg Bundesratsinitiativen mangels notwendiger Koordination mit Koalitionspartnern vereinfachten. 


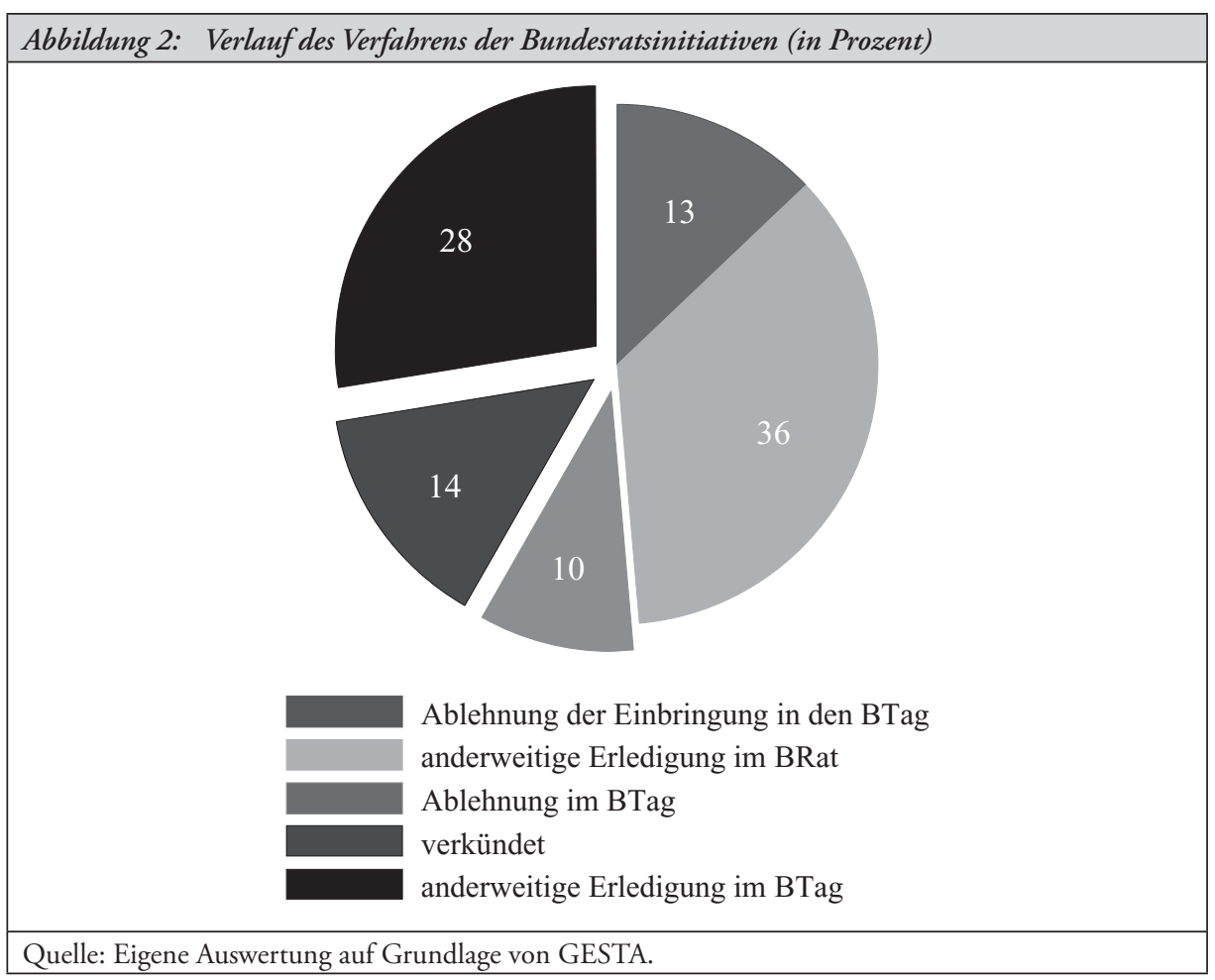

\subsection{Operationalisierung der Mehrheitsverhältnisse und Initiativkonfigurationen}

Es ist die zentrale analytische Crux zu unterscheiden, ob die Akteure im Bundesrat von den Interessen ihrer Länder geleitet sind oder nach der Logik des Parteienwettbewerbs auf Bundesebene handeln. ${ }^{24} \mathrm{Da}$ es praktisch unmöglich ist, die Motivlage der Landesvertreter direkt zu erfassen ${ }^{25}$, muss ein indirekter Nachweis geführt werden. Um einen potentiellen Einfluss der Mehrheitsverhältnisse auf die Initiativtätigkeit aufzudecken, werden drei verschiedene Mehrheitskonfigurationen unterschieden ${ }^{26}$ : Regierungsmehrheit, Oppositionsmehrheit und keine eindeutige Mehrheit. Im Einzelnen bedeutet dies, dass bei einer Regie-

24 Siehe für eine innovative analytische Unterscheidung Thomas Bräuninger / Thomas Gschwend / Susumu Shikano, a.a.O. (Fn. 8).

25 Vgl. Steffen Ganghof, Promises and Pitfalls of Veto Player Analysis, in: Swiss Political Science Review, 9. Jg. (2003), H. 2, S. $1-25$.

26 Vgl. Sven Leunig, $\mathrm{AB}(\mathrm{C})$ oder „ROM“? Zur Operationalisierung von Mehrheitsverhältnissen im Bundesrat, in: ZParl, 37. Jg. (2006), H. 2, S. 402 - 420; Michael F. Feldkamp, Datenhandbuch zur Geschichte des Deutschen Bundestages 1994-2003, Baden-Baden 2005; Matthias Lehnert I Eric Linhart, Der Einfluss der Mehrheitsverhältnisse im Vermittlungsausschuss auf die deutsche Gesetzgebung, in: Susumu Shikano / Joachim Behnke / Thomas Bräuninger (Hrsg.), Jahrbuch für Handlungs- und Entscheidungstheorie, Wiesbaden 2009, S. 149 - 179; Wenke Seemann, Do State Elections Affect Federal Governments' Legislative Behaviour? Empirical Evidence from the German Case, 1976-2005, in: German Politics, 17. Jg. (2008), H. 3, S. 252 - 269. 
rungsmehrheit im Bundesrat jene Länder über eine absolute Stimmenmehrheit verfügen, deren Landesregierungen mit der im Bund regierenden Koalition übereinstimmen beziehungsweise deren Landesregierungen ausschließlich von einer der beiden regierenden Koalitionsparteien gestellt werden. Bei einer Oppositionsmehrheit im Bundesrat besitzen jene Länder eine absolute Mehrheit der Stimmen, deren Landesregierung ausschließlich aus einer Partei oder Parteien besteht, die im Bundestag die Opposition stellen. Keine eindeutige Mehrheit im Bundesrat liegt dann vor, wenn weder die Ländergruppen, deren Regierungen ausschließlich aus einer Partei oder Parteien der Regierungskoalition im Bund bestehen, noch jene, deren Regierungen ausschließlich aus einer Partei oder Parteien der Opposition im Bund bestehen, eine eindeutige Mehrheit im Bundesrat erreichen.

Wie diese unterschiedlichen Mehrheitsverhältnisse auf die Initiativtätigkeit des Bundesrates wirken, wird erfasst, indem die antragstellenden Länder und Ländergruppen genauer anhand der parteipolitischen Kategorisierung differenziert werden. Zu diesem Zweck wird das ROMÜ-Modell als eine substantielle Ergänzung des ROM-Modells genutzt. Diese Ergänzung wurde notwendig, da bestehende Modelle konzipiert wurden, um die Abstimmung von Bundestagsvorlagen im Bundesrat zu untersuchen. Für eine trennscharfe Betrachtung der Länderinitiativtätigkeit ist eine Unterscheidung in vier Konfigurationen sinnvoll:

- R-Initiativen: Initiativen von einem Land oder Ländergruppen, deren Landesregierung sich ausschließlich aus einer Partei oder Parteien zusammensetzt, die auf Bundesebene die Regierung bilden.

- O-Initiativen: Initiativen von einem Land oder Ländergruppen, deren Landesregierung sich ausschließlich aus einer Partei oder Parteien zusammensetzt, die auf Bundesebene die Opposition stellen oder auf Bundesebene nicht vertreten sind.

- M-Initiativen: Initiativen von einem Land oder Ländergruppen, deren Regierung sich ausschließlich aus Parteien zusammensetzt, die auf Bundesebene einerseits der Regierung, andererseits der Opposition angehören. Auch Kombinationen aus R- oder O-Ländern mit M-Ländern zählen zu dieser Initiativkonfiguration.

- Ü-Initiativen: Initiativen von Ländergruppen, an denen mindestens eine Landesregierung beteiligt ist, die sich ausschließlich aus einer Partei oder Parteien zusammensetzt, die auf Bundesebene die Regierung stellen und an denen mindestens eine Landesregierung beteiligt ist, die sich ausschließlich aus einer Partei oder Parteien zusammensetzt, die auf Bundesebene die Opposition stellen oder auf Bundesebene nicht vertreten sind. ${ }^{27}$

Um zwischen den widerstreitenden Handlungslogiken zu unterscheiden, sind M- und ÜInitiativen besonders aufschlussreich. Für das Zustandekommen von M-Initiativen ist es mindestens notwendig, dass in einem Land Interessen bestehen, die quer zur bundespolitischen Machtkonstellation verlaufen. Ü-Initiativen können als leichte Steigerung davon begriffen werden, da hier mindestens zwei verschiedene Länder ein gemeinsames, parteipolitisch neutrales Interesse formulieren. ${ }^{28}$ In einem vollständig parteipolitisierten Bundesrat wären diese Initiativen praktisch ausgeschlossen. Ihr Vorhandensein erlaubt es daher einzuschätzen, inwiefern im Bundesrat Länderinteressen vertreten werden.

27 Zusätzlich kann auch eine gemischte Landesregierung an diesen Initiativen beteiligt sein.

28 Dies ist streng genommen auch bei M-Initiativen mehrerer Länder der Fall. 


\subsection{Einfluss der Mehrheitsverhältnisse auf die Initiativkonfigurationen}

Tabelle 1 fasst gegliedert nach Mehrheitsverhältnissen zusammen, welchen Anteil die nach dem ROMÜ-Schema klassifizierten Länderinitiativen an den insgesamt 685 Bundesratsvorlagen haben, die an den Bundestag weitergeleitet wurden. Die Tabelle spiegelt damit das Verhalten des Bundesrates als Kollektivorgan wider. Mit Hinblick auf die Anteile von Rund O-Initiativen lässt sich ein deutlicher Einfluss der Mehrheitsverhältnisse ausmachen. In Phasen einer kongruenten Mehrheit in Bundesrat und Bundestag dominierten Initiativen aus Regierungsländern mit 54 Prozent gegenüber 22 Prozent aus Oppositionsländern. Diese Verhältnisse waren im Fall eines inkongruenten Bundesrates nahezu umgekehrt. Nur noch 10,5 Prozent der erfolgreichen Ländervorlagen stammten aus R-Ländern, für ganze 62 Prozent zeichneten oppositionsregierte Bundesländer verantwortlich. Diese Ergebnisse bestätigen die Vermutung, dass der Bundesrat bei günstigen Mehrheitsverhältnissen von der Opposition als Initiativinstrument genutzt wird. Durchschnittlich knapp 30 Prozent der Gesetzesentwürfe wurden von M- und Ü-Ländern verfasst. Bei einer gemischten Mehrheit stieg dieser Anteil auf 37 Prozent. Insgesamt unterliegen die Anteile solcher Initiativen bei Veränderung der Mehrheitsverhältnisse aber wesentlich geringeren Schwankungen.

Der Quotient aus den im Bundesrat verabschiedeten Vorlagen (685) und der Gesamtzahl der von einzelnen Ländern eingebrachten Initiativen (1.332) kann als Erfolgsquote bezeichnet werden. ${ }^{29}$ Diese Quoten sind in Tabelle 2 nach Initiativkonfiguration und Mehrheitssituation aufgeschlüsselt. Die durchschnittliche Erfolgsquote von 51 Prozent besagt also, dass nur gut die Hälfte aller in den Bundesrat eingebrachten Länderinitiativen letztlich zu einer Gesetzesvorlage des Bundesrates führten. Dies unterstreicht, dass Gesetzesinitiativen - im Hinblick auf Landes- oder Bundespolitik - „auch dann als öffentlichkeitswirksames Mittel der Politik angesehen werden, wenn sie nicht die erforderliche Mehrheit im Bundesrat finden" 30 .

\begin{tabular}{|l|c|c|c|c|c|}
\hline Tabelle 1: Verteilung von ROMÜ-Initiativen nach parteipolitischen Mehrheitsverhältnissen \\
im Bundesrat (Prozentwerte in Klammern)
\end{tabular}

29 Die Mehrheitsverhältnisse haben keinen Einfluss auf den Anteil anderweitig erledigter Bundesratsvorlagen, so dass davon keine Verzerrung der Erfolgsquote zu erwarten ist.

30 Wolfgang Ismayr, a.a.O. (Fn. 13), S. 394. 


\begin{tabular}{|l|c|c|c|c|c|}
\hline \multicolumn{6}{|c|}{ Tabelle 2: Erfolgsquote von Länderinitiativen nach Mehrheitsverhältnissen (in Prozent) } \\
\hline & $\mathrm{R}$ & $\mathrm{O}$ & $\mathrm{M}$ & Ü & $\varnothing$ \\
\hline R-Mehrheit & 53 & 25 & 66 & 93 & 59 \\
O-Mehrheit & 30 & 55 & 68 & 83 & 59 \\
M-Mehrheit & 64 & 42 & 57 & 100 & 66 \\
$\varnothing$ & 49 & 41 & 64 & 92 & 61 \\
\hline Anmerkung: Vgl. Erläuterungen in Tabelle 1. \\
Quelle: Eigene Auswertung auf Grundlage von GESTA.
\end{tabular}

Wenn auch weniger deutlich als bei den Anteilen in Tabelle 1, schwanken die Erfolgsquoten von R- und O-Initiativen in Abhängigkeit der Mehrheitsverhältnisse. ${ }^{31} 53$ Prozent aller in den Bundesrat eingebrachten R-Länder-Vorlagen beziehungsweise 55 Prozent aller OLänder-Vorlagen wurden unter kongruenten respektive inkongruenten Mehrheitsverhältnissen an den Bundestag weitergeleitet. Dagegen erreichten nur 25 Prozent der O-Vorlagen und 30 Prozent der R-Vorlagen unter jeweils ungünstigen Mehrheitsverhältnissen den Bundestag. Zusätzlich tritt ein interessantes Muster zu Tage: Die Erfolgsaussichten einer Vorlage aus einer M-Ländergruppe und insbesondere aus einer Ü-Ländergruppe liegen mit 64 Prozent, respektive 92 Prozent weit über dem Durchschnitt. Dass diese Quoten zudem von Veränderungen in den Mehrheitsverhältnissen relativ unberührt bleiben, lässt vermuten, dass genuine Länderinteressen einen geringeren, aber soliden Teil der Initiativtätigkeit des Bundesrates bestimmen.

\section{Gesetzesinitiativen im Bundesrat: Parteipolitik, aber auch Länderinteressen}

Die parteipolitischen Mehrheitsverhältnisse im Bundesrat entfalten also einen deutlichen Einfluss auf die Initiativtätigkeit des Bundesrates. Unter einer oppositionellen Mehrheit stammen 62 Prozent der an den Bundestag weitergeleiteten Bundesratsinitiativen aus der Feder von O-Ländern. Im umgekehrten Fall gehen 54 Prozent der Vorlagen auf R-Länder zurück. Dieses Muster spricht dafür, dass sich parteipolitische Konflikte auch im Initiativverhalten des Bundesrates niederschlagen. Insbesondere die Opposition nutzt die Ländervertretung, um ihre Vorstellungen in den Gesetzgebungsprozess einzubringen. Da ihr auch im Bundestag selbst ein Initiativrecht zusteht, ist anzunehmen, dass der Umweg über den Bundesrat genutzt wird, um den Entwürfen einen vermeintlich parteipolitisch neutralen Anstrich zu verleihen. Zudem ist denkbar, dass oppositionelle Entwürfe von einem größeren öffentlichen Interesse profitieren, wenn sie bereits die formale Bestätigung durch das Verfassungsorgan Bundesrat erfahren haben. Dieser Umstand dürfte den Bundesrat insbesondere für ambitionierte Ministerpräsidenten zu einem wichtigen Forum machen. Interessanterweise brachte Bayern zwischen 1998 und 2002 überdurchschnittliche viele Vorlagen in den Bundesrat ein. Es stellt sich die Frage, ob der damalige Ministerpräsident und Kanzlerkandidat Edmund Stoiber diesen Weg nutzte, um seinen bundespolitischen Anspruch zu unterstreichen.

31 Dabei ist zu bedenken, dass der Erfolgsquotient zum Teil die antizipierende Reaktion auf die Mehrheitsverhältnisse im Bundesrat beinhalten dürfte. 
Über den klaren Gegensatz zwischen O- und R-Ländern hinaus werden in geringerem Umfang auch Initiativen von Ländergruppen gestartet, deren Regierungsparteien sich im Bund als Regierung und Opposition und somit als Kontrahenten gegenüberstehen. Dass diese überparteilichen Initiativen überhaupt zustande kommen und ihre hohe Erfolgsquote von 78 Prozent lässt vermuten, dass bei aller Parteipolitik auch in bescheidenem Maße sachpolitische Länderinteressen in die Gesetzesvorlagen des Bundesrates Eingang finden. In diesem Sinne beantwortet unsere Untersuchung die Ausgangsfrage, ähnlich wie andere Analy$\operatorname{sen}^{32}$, mit einem vorsichtigen „sowohl als auch“. Für eine sicherere und differenziertere Interpretation dieser Ergebnisse sind weitere Studien wünschenswert. ${ }^{33}$

32 Vgl. Thomas Bräuninger/ Thomas Gschwend/Susumu Shikano, a.a.O. (Fn. 8), S. 227.

33 Qualitativ verspricht eine Inhaltsanalyse einzelner Gesetzesvorlagen, insbesondere der M- und Ü-Initiativen näheren Aufschluss. Quantitativ erscheint die getrennte Untersuchung nach Politikfeldern sinnvoll. Burkhart und Manow zeigen beispielsweise, dass die Konfliktintensität bei Finanzen sowie im Politikfeld Arbeit und Soziales besonders hoch ist. Vgl. Simone Burkhart I Philip Manow, a.a.O. (Fn. 22).

\section{Komplexe Koalitionen: Welchen Nutzen bringen sie den Parteien?}

\section{Stephan Klecha}

Mit der Etablierung des „fluiden Fünfparteiensystems“1 in Ost- und Westdeutschland sind Regierungsbildungen komplexer geworden. Mehrheiten von einer oder zwei Parteien im Anschluss an Wahlen sind keineswegs mehr sicher. Spätestens die Bundestagswahl 2005 und die Landtagswahlen in Hessen, im Saarland, in Thüringen sowie zuletzt in NordrheinWestfalen haben deutlich gemacht, dass Mehrheiten abseits Großer Koalitionen vielfach nur auf Basis komplexer Koalitionen möglich sind. Darunter werden im Folgenden alle Bündnisse verstanden, in denen mehr als zwei miteinander in Wahlen konkurrierende Parteien auf legislativer oder exekutiver Ebene mit Blick auf die Erlangung von Regierungsmacht oder die Durchsetzung von Regierungshandeln zusammenwirken. Diese Begriffsbestimmung umfasst somit alle formalen Koalitionen sowie die Fälle tolerierter Minderheitsregierungen, die sich im Parlament auf mindestens drei Fraktionen stützen. Mangels Konkurrenz bei den Wahlen zueinander und wegen der Fraktionsgemeinschaft im Deutschen Bundestag werden CDU und CSU hierbei als eine Partei verstanden.

1 Oskar Niedermayer, Nach der Vereinigung: Der Trend zum fluiden Fünfparteiensystem, in: Oscar W. Gabriel / ders. / Richard Stöss (Hrsg.), Parteiendemokratie in Deutschland, Bonn 2001, S. 107 -127 . 